\title{
Влияние электрического поля на соотношение параметров Рашба и Дрессельхауза в гетероструктурах $A^{\prime \prime \prime} B^{V}$
}

\author{
(C) В.Е. Дегтярев, С.В. Хазанова "А.А. Конаков \\ Национальный исследовательский Нижегородский государственный университет им. Н.И. Лобачевского, \\ 603950 Нижний Новгород, Россия \\ ๑E-mail: khazanova@phys.unn.ru
}

(Получена 27 апреля 2017 г. Принята к печати 12 мая 2017 г.)

С помощью 8-зонной модели Кейна и конечно-разностной схемы с дискретизацией в координатном пространстве численно выполнены расчеты энергий подзон размерного квантования и огибающих волновых функций для квантовых ям [001] на основе полупроводников $\mathrm{A}^{\mathrm{III}} \mathrm{B}^{\mathrm{V}}$ со структурой цинковой обманки. Исследовано влияние зонных параметров квантовой ямы, а также величины внешнего электрического поля, ориентированного вдоль направления роста структуры, на соотношение параметров спин-орбитального взаимодействия Рашба и Дрессельхауза. Показано, что в структурах $\mathrm{GaAs} / \mathrm{InGaAs}$ при определенных значениях электрического поля возможно равенство параметров спин-орбитального взаимодействия, что является условием формирования устойчивых спиновых „хеликсов“. Установлено также, что в симметричных ямах $\mathrm{GaAs} / \mathrm{InGaAs}$ при определенных ширинах ям и химическом составе барьеров может исчезать линейное по волновому вектору спин-орбитальное взаимодействие.

DOI: 10.21883/FTP.2017.11.45091.05

\section{1. Введение}

Благодаря более ярко выраженным спин-зависимым явлениям квантово-размерные структуры все чаще используются при проектировании приборов спинтроники. Спин-орбитальное взаимодействие (СОВ) приводит к перестройке энергетического спектра низкоразмерных систем, а также является причиной множества физических явлений, таких как спин-гальванический эффект [1], спиновый эффект Холла [2], спиновая релаксация [3], являющихся ключевыми для построения приборов на спин-зависимых эффектах. Наличие в гетероструктурах тяжелых элементов и асимметрии потенциала может приводить к еще более заметным спиновым явлениям и необходимости их подробного изучения.

В двумерных кристаллических системах основной вклад в СОВ вносят взаимодействие Дрессельхауза, обусловленное асимметрией элементарной ячейки опорного кристалла, и взаимодействие Рашба, вызванное асимметрией гетероструктуры. Последнее может эффективно управляться внешним электрическим полем [4]. Такая модификация параметров СОВ внешним полем существенным образом влияет на кинетические и динамические спиновые характеристики двумерного электронного газа. В частности, в наиболее распространенном случае $\mathrm{A}^{\mathrm{III}} \mathrm{B}^{\mathrm{V}}$ квантовых ям (КЯ) с направлением роста [001] при определенных соотношениях между параметрами СОВ возможна реализация спиновой $\mathrm{SU}(2)$ симметрии [5]. В таких КЯ заметно снижается скорость спиновой релаксации электронов проводимости [6], что расширяет возможности проектирования спиновых приборов на их основе.

Необходимо отметить, что экспериментальное определение параметров СОВ в каждом случае остается сложной задачей ввиду необходимости применения спе- циальных методов исследования. В связи с этим для прогнозирования спиновых свойств необходим предварительный дизайн квантово-размерных гетероструктур с учетом СОВ. Модельные расчеты позволяют проследить корреляцию между технологическими параметрами роста и величиной СОВ в структурах с произвольным составом, оценить расщепление энергетических подзон, а также исследовать возможность управления соотношением параметров СОВ в различных гетеросистемах.

В данной работе путем непосредственного численного моделирования мы предлагаем методику, позволяющую оценить параметр Дрессельхауза для симметричных квантово-размерных структур и количественно определить вклад Рашба в СОВ в присутствии асимметрии структуры, вызванной электрическим полем, приложенным в направлении ее роста. Для гетероструктур $\mathrm{In}_{x} \mathrm{Ga}_{1-x} \mathrm{As} / \mathrm{GaAs}$ и $\operatorname{In}_{x} \mathrm{Ga}_{1-x} \mathrm{Sb} / \mathrm{GaSb}$ изучаются зависимости параметров спинового расщепления от электрического поля для различных подзон размерного квантования и ширин КЯ. Кроме того, нашей задачей является выявление факторов, влияющих на соотношение между параметрами Рашба и Дрессельхауза, определяющих степень и характер спин-зависимых явлений в исследуемых структурах.

\section{2. Метод расчета}

В работе теоретически исследуются электронные состояния в КЯ $\operatorname{In}_{x} \mathrm{Ga}_{1-x} \mathrm{As} / \mathrm{GaAs}$ при учете COB. Как известно, с точностью до членов 3-го порядка по волновому вектору электронный гамильтониан вблизи дна зоны проводимости объемного кристалла со структурой 
цинковой обманки имеет вид [7]

$$
\hat{H}=\frac{\hbar^{2} \mathbf{k}^{2}}{2 m} \hat{\sigma}_{0}+\gamma_{D}\left(\kappa_{x} \hat{\sigma}_{x}+\kappa_{y} \hat{\sigma}_{y}+\kappa_{z} \hat{\sigma}_{z}\right),
$$

где $\quad \kappa_{x}=k_{x}\left(k_{y}^{2}-k_{z}^{2}\right), \quad \kappa_{y}=k_{y}\left(k_{z}^{2}-k_{x}^{2}\right) \quad$ и $\quad \kappa_{z}=$ $=k_{z}\left(k_{x}^{2}-k_{y}^{2}\right)-$ компоненты вектора, характеризующего эффективное магнитное поле, обусловленное $\mathrm{COB}, k_{x}, k_{y}$ и $k_{z}-$ компоненты волнового вектора электрона, $\hat{\sigma}_{x}, \hat{\sigma}_{y}, \hat{\sigma}_{z}$ и $\hat{\sigma}_{0}-$ матрицы Паули и единичная матрица соответственно, $\gamma_{D}$ - параметр Дрессельхауза в объемном материале, а оси декартовых координат $x, y$ и $z$ отвечают кристаллографическим направлениям [001]. Второе слагаемое в выражении (1) называют СОВ Дрессельхауза в объемном материале, оно обусловлено отсутствием центра инверсии в элементарной ячейке кристалла.

В КЯ СОВ претерпевает изменение вследствие размерного квантования для носителей в направлении роста ямы. Считая, что спектр квантуется в направлении а, электронные состояния в КЯ, отвечающие малым двумерным волновым векторам, будут описываться двухкомпонентной (с учетом спина) огибающей функцией $\psi_{n, s}(\mathbf{a} \cdot \mathbf{r})$, где $n-$ номер подзоны размерного квантования, а индекс $s$ характеризует спиновое состояние. Далее будем полагать, что КЯ выращены в кристаллографическом направлении [001], которому в гамильтониане (1) отвечает ось $z$.

Перепишем гамильтониан СОВ для трехмерного кристалла в виде

$$
\begin{aligned}
\hat{H}_{3 D}= & \gamma_{D} k_{z} \hat{\sigma}_{z}\left(k_{x}^{2}-k_{y}^{2}\right)-\gamma_{D} k_{z}^{2}\left(k_{x} \hat{\sigma}_{x}-k_{y} \hat{\sigma}_{y}\right) \\
& +\gamma_{D}\left(k_{x} k_{y}^{2} \hat{\sigma}_{x}-k_{y} k_{x}^{2} \hat{\sigma}_{y}\right) .
\end{aligned}
$$

В направлении роста ямы вклады в гамильтониан заменяются на дифференциальные операторы согласно процедуре симметризации [8]:

$$
\begin{gathered}
k_{z} \rightarrow-i \frac{\partial}{\partial z}, \\
\gamma_{D} k_{z} \rightarrow-\frac{i}{2}\left(\frac{\partial}{\partial z} \gamma_{D}(z)+\gamma_{D}(z) \frac{\partial}{\partial z}\right), \\
-\gamma_{D} k_{z}^{2} \rightarrow \frac{\partial}{\partial z} \gamma_{D}(z) \frac{\partial}{\partial z},
\end{gathered}
$$

откуда, усредняя для $n$-й подзоны размерного квантования в направлении роста ямы и учитывая, что среднее от первого слагаемого в гамильтониане (2) по состоянию с огибающей $\psi_{n, s}(z)$ равно нулю [9], получим [10]

$$
\hat{H}_{2 D}^{(n)}=\beta_{n}\left(k_{x} \hat{\sigma}_{x}-k_{y} \hat{\sigma}_{y}\right)+\gamma_{D}^{(n)}\left(k_{x} k_{y}^{2} \hat{\sigma}_{x}-k_{y} k_{x}^{2} \hat{\sigma}_{y}\right),
$$

где

$$
\beta_{n}=\left\langle\frac{\partial}{\partial z} \gamma_{D}(z) \frac{\partial}{\partial z}\right\rangle_{n}
$$

- параметр Дрессельхауза для $n$-й подзоны в КЯ, $\gamma_{D}^{(n)}=\left\langle\gamma_{D}(z)\right\rangle_{n}$, а символ $\langle\ldots\rangle_{n}$ означает усреднение по $n$-й подзоне размерного квантования. В результате в КЯ спин-орбитальный вклад в гамильтониан преобразуется за счет эффекта размерного квантования и содержит теперь как линейные, так и кубические по волновому вектору вклады.

Наличие асимметрии профиля потенциала КЯ приводит к появлению линейного по волновому вектору спинорбитального вклада Рашба в электронный гамильтониан [11]:

$$
\hat{H}_{R}^{(n)}=\alpha_{n}\left(k_{y} \hat{\sigma}_{x}-k_{x} \hat{\sigma}_{y}\right),
$$

где $\alpha_{n}$ - параметр Рашба для $n$-й подзоны размерного квантования. Простейшим способом введения асимметрии структуры является помещение ее во внешнее электрическое поле, ориентированное в направлении роста КЯ. В этом случае параметр Рашба для каждой подзоны прямо пропорционально зависит от приложенного поля [11].

Пренебрегая интерфейсными вкладами в СОВ [12,13], а также членами 3-го порядка по волновому вектору в (4) [10], в асимметричной КЯ на основе полупроводниковых соединений со структурой цинковой обманки допустимо 2 вклада в СОВ (Рашба и Дрессельхауза), а эффективный электронный гамильтониан в $n$-ой подзоне принимает вид

$$
\begin{aligned}
\hat{H}_{n}\left(k_{x}, k_{y}\right)= & \frac{\hbar^{2}\left(k_{x}^{2}+k_{y}^{2}\right)}{2 m} \hat{\sigma}_{0}+\alpha_{n}\left(k_{y} \hat{\sigma}_{x}-k_{x} \hat{\sigma}_{y}\right) \\
& +\beta_{n}\left(k_{x} \hat{\sigma}_{x}-k_{y} \hat{\sigma}_{y}\right) .
\end{aligned}
$$

Метод, использованный для получения гамильтониана (6), применим лишь в области с малыми волновыми векторами вблизи дна зоны проводимости опорного кристалла, так что хорошо описывает дисперсию лишь в нижней подзоне размерного квантования [14]. Для расчета параметров СОВ в нескольких подзонах размерного квантования более корректным является применение многозонных моделей, точно учитывающих взаимодействие зон. В КЯ на основе твердого раствора InGaAs ширина запрещенной зоны оказывается заметно меньше, чем энергетический зазор между первой и второй зонами проводимости, в связи с чем расчет параметров СОВ возможен в рамках 8-зонной модели Кейна $[15,16]$, точно учитывающей $\mathbf{k} \cdot \mathbf{p}$-взаимодействие зоны проводимости и валентной зоны, а влияние всех остальных зон - по теории возмущений. С использованием этого подхода энергетический спектр гетероструктуры и соответствующие 8-компонентные огибающие $\Psi_{n, s}\left(k_{x}, k_{y}, z\right)$ волновых функций могут быть найдены из решения уравнения шредингеровского типа:

$$
\begin{aligned}
\left(\hat{H}_{8 \cdot 8}\left(k_{x}, k_{y},-i \frac{\partial}{\partial z}\right)\right. & \left.+\hat{I}_{8.8} V(z)\right) \Psi_{n, s}\left(k_{x}, k_{y}, z\right) \\
& =E_{n, s}\left(k_{x}, k_{y}\right) \Psi_{n, s}\left(k_{x}, k_{y}, z\right),
\end{aligned}
$$

где $\hat{H}_{8.8}\left(k_{x}, k_{y}, z\right)$ - гамильтониан Кейна [15], $V(z)-$ электростатический потенциал структуры, учитываю- 
щий ее асимметрию, а $E_{n, s}\left(k_{x}, k_{y}\right)$ - энергия размерного квантования состояния с двумерным волновым вектором со спином $s$ в $n$-й подзоне размерного квантования.

Параметры гамильтониана Кейна для твердых растворов $\operatorname{In}_{x} \mathrm{Ga}_{1-x}$ As с точностью до квадратичных по составу $x$ вкладов взяты из работы [16]. Параметры Латтинжера преобразованы в параметры Дрессельхауза-КипаКиттеля и затем скорректированы для использования их в гамильтониане Кейна. Для решения проблемы появления быстро осциллирующих нефизических решений [17] квадратичный по $\mathbf{k}$ член гамильтониана для зоны проводимости был принят равным $\hbar^{2} \mathbf{k}^{2} / 2 m_{0}$, а межзонный матричный элемент импульса $P$ определялся из значения эффективной массы для зоны проводимости [18].

Для непосредственного численного решения уравнения (7) использовался метод конечных разностей. В рамках данного метода параметры гамильтониана (обозначим их условно $A(z))$ и огибающие волновых функций задаются на одномерной равномерной дискретной сетке в направлении роста:

$$
\begin{gathered}
A(z) \rightarrow A\left(z_{0}+l d z\right) \equiv A_{l}, \\
\Psi\left(k_{x}, k_{y}, z\right) \rightarrow \Psi_{l}\left(k_{z}, k_{y}, z_{0}+l d z\right),
\end{gathered}
$$

где $d z-$ шаг дискретизации, $z_{0}$ - координата нулевого узла. При этом рассматриваемые дифференциальные операторы (3) преобразуются к матричным [19]. Подставляя преобразованные операторы в (7), мы приходим к задаче о собственных значениях матрицы [20], зависящей от двумерного волнового вектора.

Дисперсионные зависимости для различных подзон размерного квантования в КЯ, получаемые в ходе численных расчетов изложенным методом, должны соответствовать собственным значениям гамильтониана (6):

$$
E_{n, s}(\mathbf{k})=\frac{\hbar^{2}}{2 m} \mathbf{k}^{2}+\frac{s}{2} \Delta E_{n}(\mathbf{k})
$$

где $\Delta E_{n}(\mathbf{k})$ - спиновое расщепление электронной подзоны размерного квантования с номером $n$, угловая зависимость которого в двумерном импульсном пространстве имеет вид

$$
\Delta E_{n}(\mathbf{k})=2 k \sqrt{\alpha_{n}^{2}+\beta_{n}^{2}+2 \alpha_{n} \beta_{n} \sin 2 \varphi},
$$

где $\varphi=\arctan \left(k_{y} / k_{x}\right)$.

Для непосредственного нахождения значений $\alpha_{n}$ и $\beta_{n}$ мы вводим линейные по $k$ коэффициенты спинового расщепления $b_{n \pm}$ вдоль соответствующих им направлений $\Sigma_{ \pm}=(1, \pm 1)$ в импульсном пространстве:

$$
\Delta E_{n}\left(k_{x}=k / \sqrt{2}, k_{y}= \pm k / \sqrt{2}\right)=2\left|\alpha_{n} \pm \beta_{n}\right| k=b_{n \pm} k .
$$

Определяя численно коэффициенты $b_{n \pm}$, значения $\alpha_{n}$ и $\beta_{n}$ для каждой подзоны размерного квантования находятся из выражения (11).

\section{3. Результаты расчетов и их обсуждение}

\section{1. Симметричная квантовая яма}

Рассмотрим одиночную симметричную КЯ $\mathrm{In}_{x} \mathrm{Ga}_{1-x} \mathrm{As} / \mathrm{GaAs}(x=0.4)$, выращенную в направлении [001], и рассчитаем параметры СОВ Дрессельхауза для каждой подзоны размерного квантования в зависимости от ширины КЯ $d$. В приближении бесконечно глубокой ямы можно записать [21]:

$$
\beta_{n}=\frac{\gamma_{D} \pi^{2} n^{2}}{d^{2}}
$$

Однако выражение (12), строго говоря, неприменимо для КЯ конечной глубины ввиду проникновения электронной волновой функции в подбарьерные области гетероструктуры. Кроме того, константа Дрессельхауза имеет неоднозначную зависимость от номера подзоны размерного квантования и ширины КЯ [22]. Численное решение уравнения (7) позволяет рассчитать параметр Дрессельхауза для разных подзон размерного квантования при различных технологических и геометрических параметрах КЯ с произвольным профилем состава и любым соотношением материалов, составляющих гетероструктуру.

На рис. 1 приведены рассчитанные зависимости модуля параметра Дрессельхауза для трех первых подзон размерного квантования от ширины КЯ. Видно, что с ростом $n$ зависимость $\beta_{n}(d)$ становится немонотонной. Более того, для третьей подзоны наблюдается смена знака параметра Дрессельхауза. Данное нетривиальное поведение $\beta_{n}(d)$, наблюдаемое в численных экспериментах, обусловлено в том числе тем фактом, что в твердых растворах $\operatorname{In}_{x} \mathrm{Ga}_{1-x} \mathrm{As}$ параметр $\gamma_{D}$ меняет не только значение, но и знак в зависимости от состава [23].

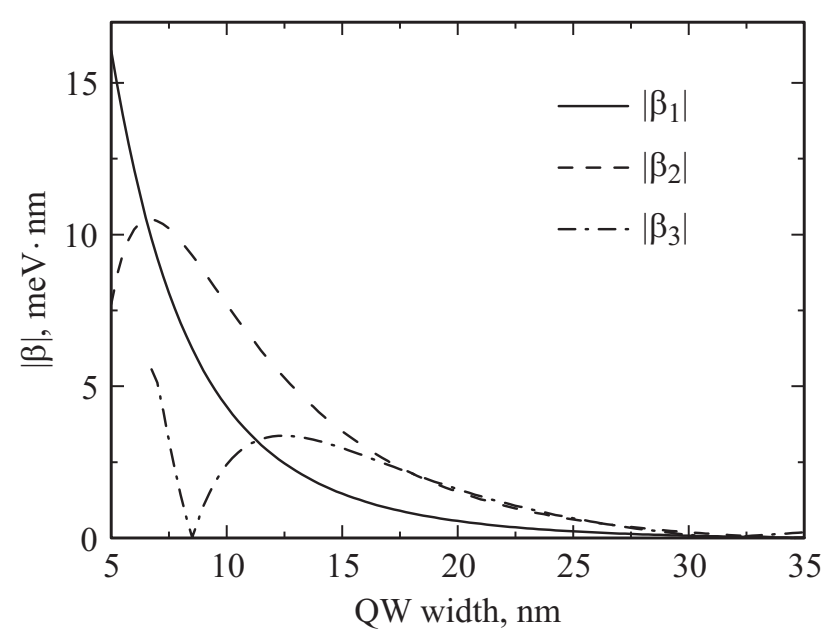

Рис. 1. Модуль параметра Дрессельхауза, рассчитанный для трех первых подзон размерного квантования в КЯ $\mathrm{In}_{x} \mathrm{Ga}_{1-x} \mathrm{As} / \mathrm{GaAs}$ в зависимости от их ширины. 


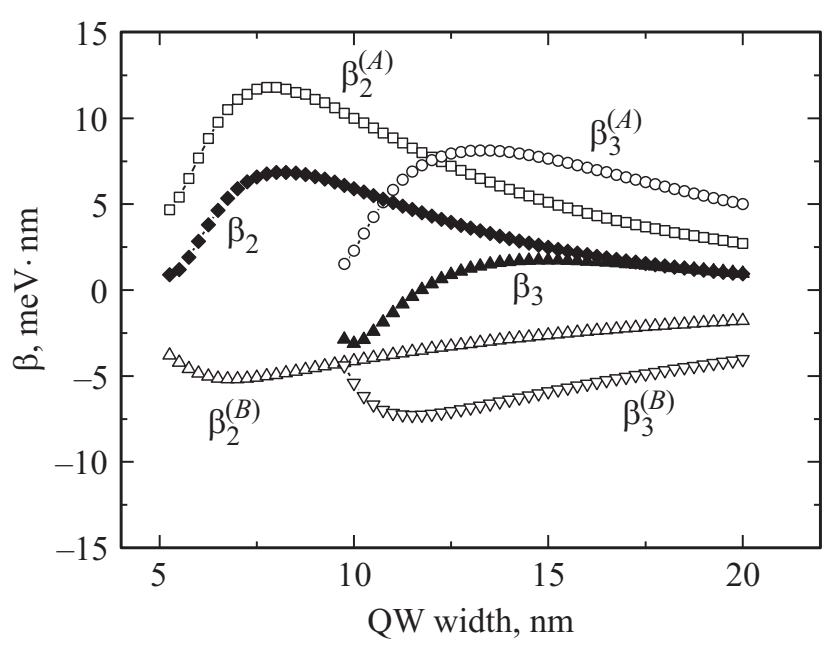

Рис. 2. Составляющие параметра Дрессельхауза в области ямы $(A)$ и барьера $(B)$ независимо, а также их суммарное значение для второй $\left(\beta_{2}\right)$ и третьей $\left(\beta_{3}\right)$ подзон размерного квантования в КЯ $\operatorname{In}_{x} \mathrm{Ga}_{1-x} \mathrm{As} / \mathrm{GaAs}$ в зависимости от их ширины.

Учитывая, что степень проникновения волновой функции в подбарьерные области меняется как с шириной ямы, так и с номером подзоны, проведем анализ функции $\beta_{n}(d)$ в рамках метода эффективной массы [24]. Для этого выражение для константы Дрессельхауза в $n$-й подзоне перепишем как

$$
\begin{gathered}
\beta_{n}=\beta_{n}^{(A)}+\beta_{n}^{(B)}, \\
\beta_{n}^{(A, B)}=\gamma_{D}^{(A, B)} \int_{A, B} \psi_{n}^{*}(z) \frac{\partial^{2}}{\partial z^{2}} \psi_{n}(z) d z,
\end{gathered}
$$

где $A-$ область квантовой ямы, $B-$ область барьеров, а огибающие $\psi_{n}(z)$ получены в рамках метода эффективной массы.

На рис. 2 представлены графики зависимости вкладов $\beta_{n}^{(A)}$ и $\beta_{n}^{(B)}$, а также их суммы $\beta_{n}$ от ширины КЯ для второй и третьей подзон размерного квантования. Как видно, даже отдельные вклады, относящиеся к области ямы или барьеров, ведут себя немонотонно с увеличением $d$. Для первой же подзоны зависимость $\beta_{n}$ от ширины КЯ не приводится в силу ее монотонности. Немонотонность вкладов $\beta_{n}^{(A, B)}$ обусловлена одновременным наложением двух эффектов: увеличением степени локализации волновой функции в области ямы и уменьшением средней кинетической энергии подзоны размерного квантования. Необходимо отметить, что зависимости суммарного значения $\beta_{n}$, приведенные на рис. 2 , качественно совпадают с поведением константы Дрессельхауза $\beta_{n}$, рассчитанной в рамках $\mathbf{k} \cdot \mathbf{p}$-метода (рис. 1). Как видно из результатов нашего анализа, постоянная Дрессельхауза в симметричных ямах $\mathrm{In}_{x} \mathrm{Ga}_{1-x} \mathrm{As} / \mathrm{GaAs}$ может носить знакопеременный характер, что при определенных значениях ширины ямы и химическом составе барьеров приводит к исчезновению линейного по волновому вектору СОВ.

\section{2. Асимметрия профиля структуры, обусловленная электрическим полем, приложенным в направлении роста ямы}

Далее мы рассматриваем асимметричную $\mathrm{In}_{x} \mathrm{Ga}_{1-x} \mathrm{As} / \mathrm{GaAs}$ КЯ шириной $d=10$ нм, в которой кроме СОВ Дрессельхауза присутствует взаимодействие Рашба. Асимметрия задается с помощью внешнего электрического поля $(E=0-0.4 \mathrm{MB} / \mathrm{Hм})$, ориентированного вдоль направления роста структуры. Решая уравнение (7), мы рассчитываем энергетический спектр гетероструктуры с учетом СОВ, описываемого суперпозицией эффектов Рашба и Дрессельхауза. На рис. 3 изображена угловая зависимость энергетического спектра нижней подзоны размерного квантования $\Delta E_{n}(\mathbf{k})$, из которой можно проследить анизотропию спинового расщепления в зависимости от напряженности электрического поля (для различных соотношений между параметрами Рашба и Дрессельхауза).

Из анализа рассчитанного спектра мы получаем значения констант Рашба и Дрессельхауза для каждой подзоны размерного квантования в структуре с КЯ $\mathrm{In}_{x} \mathrm{Ga}_{1-x} \mathrm{As} / \mathrm{GaAs}$ при различных значениях приложенного поля (рис. 4). Видно, что зависимость параметров $\alpha_{n}$ от напряженности электрического поля носит практически линейный характер. Параметр Дрессельхауза в свою очередь с увеличением внешнего поля остается практически неизменным и определяется только номером подзоны размерного квантования. Кроме того, рис. 4 наглядно демонстрирует, что для всех трех подзон

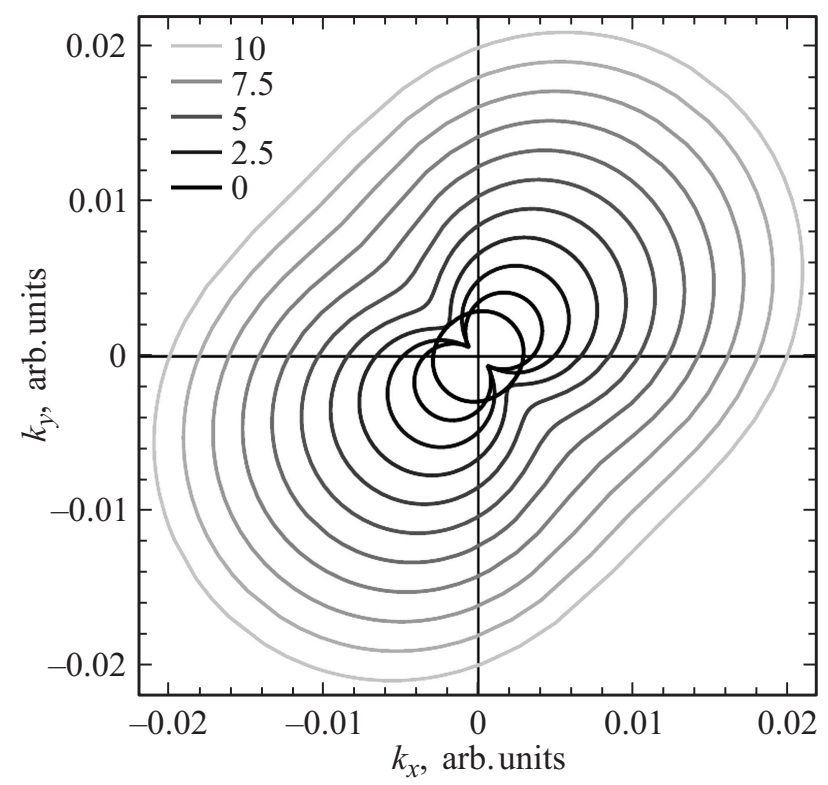

Рис. 3. Изоэнергетические поверхности 2D электронного газа с СОВ при различных значениях приложенного электрического поля (при различных соотношениях $\alpha$ и $\beta$ ). 


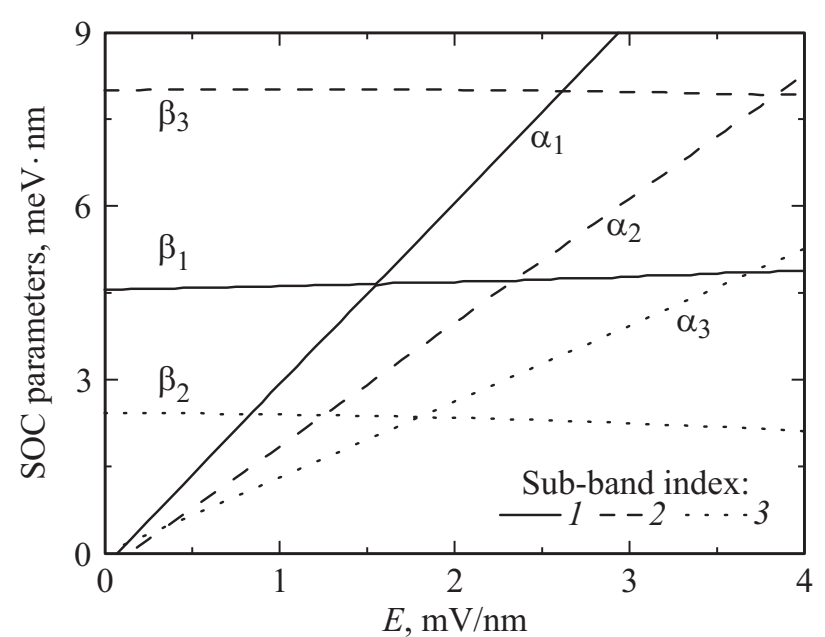

Рис. 4. Зависимость параметров Рашба и Дрессельхауза $\left(\alpha_{n}\right.$ и $\beta_{n}$ ) от напряженности электрического поля для трех первых подзон размерного квантования в КЯ $\operatorname{In}_{x} \mathrm{Ga}_{1-x} \mathrm{As} / \mathrm{GaAs} \mathrm{c}$ шириной $d=10$ нм.

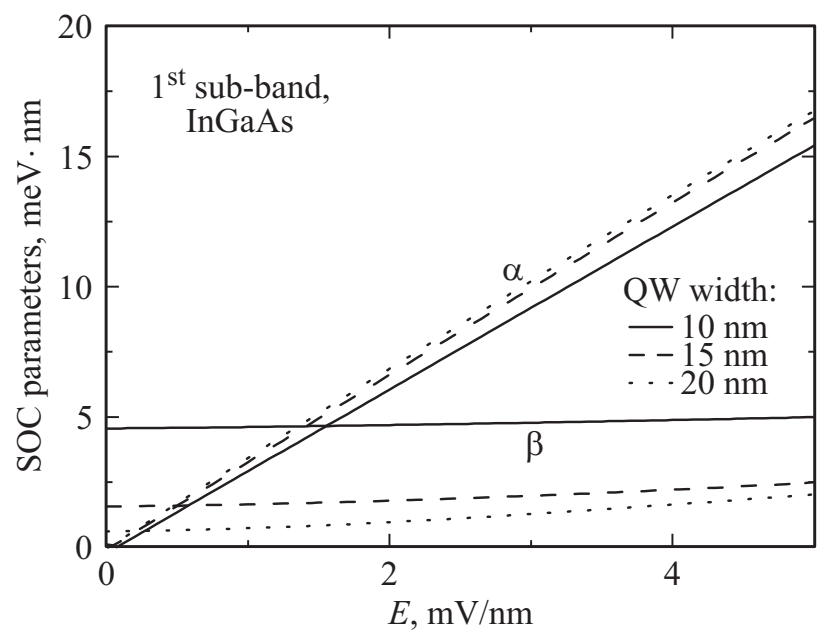

Pис. 5. Зависимость параметров СОВ от напряженности электрического поля для первой подзоны размерного квантования в КЯ $\operatorname{In}_{x} \mathrm{Ga}_{1-x} \mathrm{As} / \mathrm{GaAs}$ с ширинами $d=10,15,20$ нм.

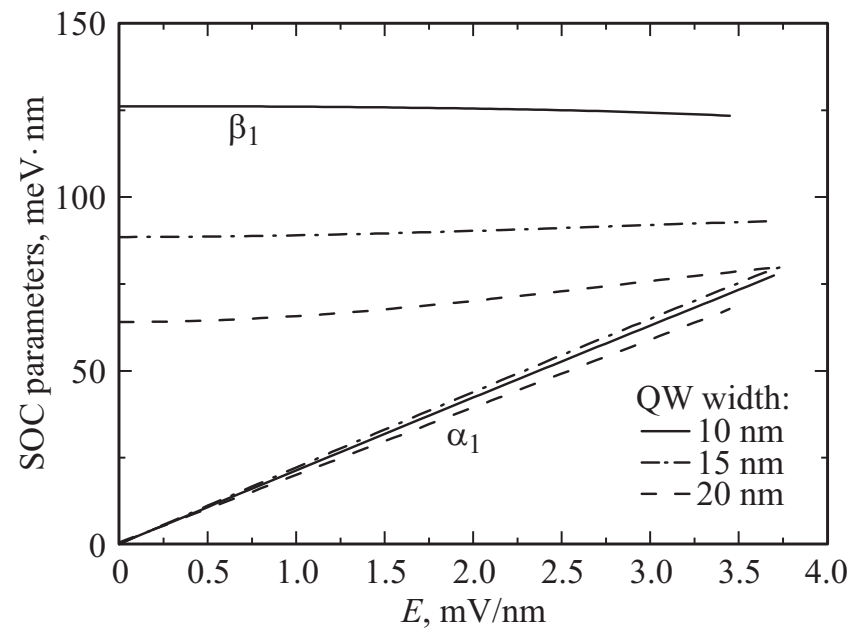

Рис. 6. Зависимость параметров СОВ от напряженности электрического поля для первой подзоны размерного квантования в КЯ $\operatorname{In}_{x} \mathrm{Ga}_{1-x} \mathrm{Sb} / \mathrm{GaSb}$ с ширинами $d=10,15,20$ нм. размерного квантования возможно равенство параметров Рашба и Дрессельхауза $\left(\alpha_{n}=\beta_{n}\right)$ при определенных значениях электрического поля, что является важным с практической точки зрения. В частности, в таких КЯ реализуется режим электронной спиновой прецессии типа устойчивого спинового „хеликса“" [5].

Также нами проведен сравнительный анализ параметров СОВ для асимметричных гетероструктур $\mathrm{In}_{x} \mathrm{Ga}_{1-x} \mathrm{As} / \mathrm{GaAs}$ и $\operatorname{In}_{x} \mathrm{Ga}_{1-x} \mathrm{Sb} / \mathrm{GaSb}(x=0.4)$ с различной шириной КЯ $(d=10-20$ нм $)$ для первой подзоны размерного квантования (рис. 5,6). В отличие от КЯ $\operatorname{In}_{x} \mathrm{Ga}_{1-x} \mathrm{As} / \mathrm{GaAs}$ в структурах $\operatorname{In}_{x} \mathrm{Ga}_{1-x} \mathrm{Sb} / \mathrm{GaSb}$ очевидно преобладание вклада Дрессельхауза в СОВ и заметное изменение константы $\beta$ с шириной КЯ для всех рассчитанных значений электрического поля (рис. 6). Константа Рашба, обусловленная асимметрией структуры, меняется в меньшем диапазоне, при этом остается почти постоянной с ростом ширины КЯ, аналогично и КЯ $\operatorname{In}_{x} \mathrm{Ga}_{1-x} \mathrm{As} / \mathrm{GaAs}$ (рис. 5). В целом величина спинового расщепления в КЯ на основе антимонидов заметно больше, что вызвано включением тяжелой сурьмы. Иначе говоря, диапазон изменения параметров СОВ в структурах $\operatorname{In}_{x} \mathrm{Ga}_{1-x} \mathrm{Sb} / \mathrm{GaSb}$ значительно выше, чем для $\operatorname{In}_{x} \mathrm{Ga}_{1-x} \mathrm{As} / \mathrm{GaAs}$. Однако, с другой стороны, при реальных напряженностях электрического поля равенство параметров Рашба и Дрессельхауза фактически недостижимо для гетероструктур на основе $\mathrm{InGaSb}$. Можно сделать вывод, что в КЯ $\operatorname{In}_{x} \mathrm{Ga}_{1-x} \mathrm{Sb} / \mathrm{GaSb}$ управление спиновыми параметрами с помощью реальных электрических полей возможно в гораздо меньшей степени.

В целом для обеих рассмотренных структур параметр $\alpha$, обусловленный структурной асимметрией, линейно растет с увеличением электрического поля и практически не чувствителен к изменению ширины ямы. Таким образом, величина параметра $\beta$ определяется номером подзоны размерного квантования и шириной КЯ, в то время как параметр $\alpha$ в большей степени контролируется приложенным полем.

\section{4. Заключение}

Путем непосредственных численных расчетов предложена методика, позволяющая оценить индивидуальный вклад СОВ Дрессельхауза и Рашба в зоне проводимости КЯ на основе полупроводников $\mathrm{A}^{\mathrm{III}} \mathrm{B}^{\mathrm{V}}$ со структурой цинковой обманки в присутствии поперечного однородного электрического поля. Расчеты, проведенные в данной работе, показывают, что асимметрия потенциала способствует увеличению спинового расщепления для всех подзон размерного квантования. Вычисленный в рамках 8-зонной модели Кейна параметр Рашба линейно зависит от приложенного электрического поля, оставаясь практически не чувствительным к ширине КЯ и номеру подзоны размерного квантования. В свою очередь параметр Дрессельхауза практически не зависит от внешнего приложенного поля, но существенно меняется 
с ростом номера подзоны и ширины ямы. Более того, он является немонотонной функцией ширины КЯ и даже может менять знак. Другими словами, при определенных условиях в симметричных КЯ может исчезать линейное по волновому вектору СОВ.

Оба параметра СОВ сильно чувствительны к типу материала. Более того, в КЯ $\operatorname{In}_{x} \mathrm{Ga}_{1-x} \mathrm{Sb} / \mathrm{GaSb}$ с тяжелой сурьмой параметр Дрессельхауза оказывается заметно больше константы Рашба. В результате в таких структурах затруднена реализация режима устойчивого спинового „хеликса“ посредством создания условий равенства констант Рашба и Дрессельхауза. В то же время нами продемонстрирована возможность эффективного управления соотношением между параметрами СOB с помощью внешнего электрического поля в гетероструктурах $\operatorname{In}_{x} \mathrm{Ga}_{1-x} \mathrm{As} / \mathrm{GaAs}$.

Результаты работы могут быть использованы для дизайна структур полупроводниковой спинтроники с требуемыми параметрами. Экспериментальное наблюдение предсказанных значений спинового расщепления возможно при температурах $\sim 4 \mathrm{~K}$, легко достижимых с помощью современных техник охлаждения, так что подобные расчеты могут быть полезны и для интерпретации экспериментальных результатов по измерению транспортных и оптических характеристик гетероструктур с сильным СОВ.

Работа поддержана Министерством образования и науки Российской Федерации в рамках проектной части государственного задания и выполнена при частичной поддержке РФФИ (гранты № 15-42-02254р_поволжье_а, 16-07-01102-a, 16-32-00683-мол_а, 16-3200712-мол_а и 16-57-51045-НИФ_а).

\section{Список литературы}

[1] S.D. Ganichev, E.L. Ivchenko, V.V. Bel'kov, S.A. Tarasenko, M. Sollinger, D. Weiss, W. Wegscheider, W. Prettl. Nature, 417, 153 (2002).

[2] J. Sinova, S.O. Valenzuela, J. Wunderlich, C.H. Back, T. Jungwirth. Rev. Mod. Phys., 87, 1213 (2015).

[3] M.W. Wu, J.H. Jiang, M.Q. Weng. Phys. Reports, 493, 61 (2010).

[4] J. Nitta, T. Akazaki, H. Takayanagi, T. Enoki. Phys. Rev. Lett., 78, 1335 (1997).

[5] B.A. Bernevig, J. Orenstein, S.-C. Zhang. Phys. Rev. Lett., 97, 236601 (2006)

[6] M.C. Lüffe, J. Kailasvuori, T.S. Nunner. Phys. Rev. B, 84, 075326 (2011)

[7] G. Dresselhaus. Phys. Rev., 100, 580 (1955).

[8] M. Ehrhardt, T. Koprucki. Multi-Band Effective Mass Approximations (Springer, Berlin-Heidelberg, 2014).

[9] P. Pffefer, W. Zawadski. Phys. Rev. B, 59, R5312 (1999).

[10] М.И. Дьяконов, В.Ю. Качоровский. ФТП, 20, 178 (1986).

[11] Ю.А. Бычков, Э.И. Рашба. Письма ЖЭТФ, 39, 66 (1984).

[12] O. Krebs, P. Voisin. Phys. Rev. Lett., 77, 1829 (1996).

[13] Ж.А. Девизорова, В.А. Волков. Письма ЖЭТФ, 98, 110 (2013).
[14] M. Kammermeier, P. Wenk, J. Schliemann. Phys. Rev. Lett., 117, 236801 (2016).

[15] E.O. Kane. Energy band theory. In: T.S. Moss, ed. Handbook on semiconductors (North Holland, Amsterdam, 1982) p. 193.

[16] I. Vurgaftman, J.R. Meyer, L.R. Ram-Mohan. J. Appl. Phys., 89, 5815 (2001).

[17] T.J. Watson, D.Z.-Y. Ting, T.C. McGilla. J. Appl. Phys., 93, 3974 (2003).

[18] G. Bastard. Wave Mechanics Applied to Semiconductor Heterostructures (Les Editions de Physique, Paris, 1988).

[19] R.G. Verpek, S. Steiger, B. Witzigmann. Phys. Rev. B, 76, 165320 (2007).

[20] С.В. Хазанова, В.Е. Дегтярев, Н.В. Малехонова, Д.А. Павлов, Н.В. Байдусь. ФТП, 49, 58 (2015).

[21] M.P. Walser, U. Siegenthaler, V. Lechner, D. Schuh, S.D. Ganichev, W. Wegscheider, G. Salis. Phys. Rev. B, 86, 195309 (2012).

[22] J. Fu, J.C. Egues. Phys. Rev. B, 91, 075408 (2015).

[23] A.N. Chantis, M. van Schlifgaarde, T. Kotani. Phys. Rev. Lett., 96, 086405 (2006).

[24] С.В. Хазанова, В.Н. Дегтярев, С.В. Тихов, Н.В. Байдусь. ФТП, 49, 53 (2015).

Редактор Г.А. Оганесян

\section{Influence of the electric field on the ratio between Rashba and Dresselhaus parameters in $A^{I I I} B^{V}$ heterostructures}

\author{
V.E. Degtyarev, S.V. Khazanova, A.A. Konakov \\ Lobachevsky University, \\ 603950 Nizhny Novgorod, Russia
}

\begin{abstract}
Using the 8-band Kane model and a finite-difference scheme with discretization in the coordinate space, we numerically performed the calculations of the size-quantized energy subbands and envelope functions for [001] quantum wells based on zincblende $\mathrm{A}^{\mathrm{III}} \mathrm{B}^{\mathrm{V}}$ semiconductors. The influence of the band parameters of the quantum well as well as the magnitude of the external electric field oriented along the direction of structure growth on the ratio between Rashba and Dresselhaus spinorbit coupling parameters is investigated. It is shown that in $\mathrm{GaAs} / \mathrm{InGaAs}$ structures at a certain electric field the spin-orbit coupling parameters can be equal, which is the condition for the formation of persistent spin helices. It has also been found that in the symmetric $\mathrm{GaAs} / \mathrm{InGaAs}$ wells linear in the wave vector spin-orbit coupling can disappear for certain values of well width and barriers chemical composition.
\end{abstract}

